\title{
Detection of Beam Induced Dipole-Mode Signals in the SLC S-Band Structures*
}

\author{
M. Seidel, C. Adolphsen, R. Assmann, D.H. Whittum \\ Stanford Linear Accelerator Center, Stanford University, Stanford, CA 94309
}

\begin{abstract}
Beam emittance dilution caused by wakefield effects is one of the important issues in the SLC linac. The detection of beam induced dipole mode signals in the Cband range could provide a direct measure of the strength of transverse wakefield kicks the beam experiences in the accelerating structures. We investigate the applicability of these microwave signals for beam steering purposes. The RF distribution system in the linac sectors 2,6 and 29 has been equipped with a simple experimental setup to observe the beam induced dipole mode signals. The paper discusses the setup, the mode-structure of the observed signals as well as experimental results from beam steering scans, obtained during the 95/96 SLC runs.
\end{abstract}

\section{MASTER}

Presented at the 1997 Particle Accelerator Conference

Vancouver, B.C., Canada, May 12-16, 1997

*Work supported by Department of Energy contract DE-AC03-76SF00515. 


\section{DISCLAMMER}

Portions of this document may be illegible in electronic image products. Images are produced from the best available original document. 


\title{
Detection of Beam Induced Dipole-Mode Signals in the SLC S-Band Structures
}

\author{
M. Seidel, C. Adolphsen, R. Assmann, D.H. Whittum \\ Stanford Linear Accelerator Center, Stanford University, CA 94309
}

\begin{abstract}
Beam emittance dilution caused by wakefield effects is one of the important issues in the SLC linac. The detection of beam induced dipole mode signals in the $\mathrm{C}$-band range could provide a direct measure of the strength of transverse wakefield kicks the beam experiences in the accelerating structures. We investigate the applicability of these microwave signals for beam steering purposes. The RF distribution system in the linac sectors 2,6 and 29 has been equipped with a simple experimental setup to observe the beam induced dipole mode signals. The paper discusses the setup, the mode-structure of the observed signals as well as experimental results from beam steering scans, obtained during the $95 / 96$ SLC runs.
\end{abstract}

\section{INTRODUCTION}

The SLC main linac consists of roughly 900 ten foot long accelerator sections which are operated at $2.856 \mathrm{GHz}$ (SBand) in $2 \pi / 3$ mode. The linac accelerates positron and electron beams, delivered by damping rings, from $1.2 \mathrm{GeV}$ injection energy to $47 \mathrm{GeV}$ final energy. The injected beams are naturally flat with normalized emittances of $\gamma \varepsilon_{x}=$ $30 \mathrm{~mm} \mathrm{mrad}, \gamma \varepsilon_{y}=3 \mathrm{~mm}$ mrad. During the acceleration process the vertical emittance grows typically by $100 \ldots 200 \%$ due to wakefield effects. The head of the bunch excites asymmetric fields in the accelerating structures, to lowest order dipole modes, that consequently deflect the tail of the bunch. Energy variations along the bunch lead to a chromatic filamentation of this $y-z$ correlated distribution and an increased projected emittance. This type of emittance growth can be avoided in principle if the beam could be precisely centered in the structures to prevent the excitation of Higher Order Modes (HOM's). For centering of the beam orbit one relies in the SLC on beam position monitor (BPM) information and on a good alignment of the BPM's with respect to the structures. A better way for beam steering is therefore to measure the beam induced dipole mode signals directly in the structures and to minimize them to reduce the wakefields. Such a structure BPM would measure an absolute beam position in the only relevant reference frame, namely with respect to the electrical center of the structure itself. The whole scheme is possible because the dipole mode signals occur at higher frequencies than the much stronger accelerating mode, which then can be suppressed by appropriate filtering.

It can be expected that the application of structure BPM's to the SLC would improve the performance of the machine as well as the efficiency of orbit steering procedures.

\section{SIGNAL ACQUISITION AND PROPERTIES}

Beam induced dipole mode signals can be observed at the standard high power input and output couplers of the SLC structures. In our experiments we accessed the signals using a Bethe Hole coupler in the waveguide network that distributes the RF power from the klystron to two or four structures in the SLC tunnel [1], with the consequence that we observe in general the superposition of signals from several structures. The very strong accelerating mode component in the waveguide network is suppressed by a WR 187 waveguide filter with a cutoff frequency of $3.16 \mathrm{GHz}$. The second harmonic is removed by an additional lowpass filter at $4.5 \mathrm{GHz}$. The bandpass-filtered signal is then analyzed either with a crystal detector, or a spectrum analyzer. Fig. 1 shows the time domain signal, obtained from the crystal after the passage of a single bunch at station 20-3c. This station differs from all others in that only one structure is connected to the klystron. From an estimated $Q \approx 7000$ one expects a ringing time of the signal of $1 \mu \mathrm{s}$, however, there are likely several reflections in the network which is not well matched at $4 \mathrm{GHz}$.

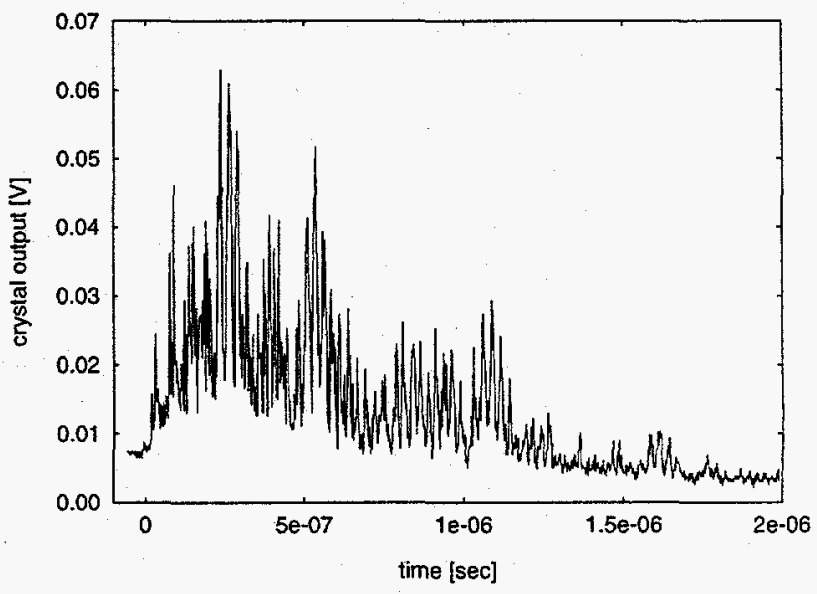

Figure 1: Crystal detector output signal showing signals in the frequency range $\approx 4-4.5 \mathrm{GHz}$. The bunch enters the structure at $t=0$.

The dipole mode spectrum extends from $4.14 \mathrm{GHz}$ to roughly $4.35 \mathrm{GHz}$. A trace of the peak-power vs. frequency that was recorded with a spectrum analyzer is shown in Fig. 2. The shown spectrum is typical for all stations that have been measured. It exhibits some features that are not expected - the signals extend in frequency below the lowest dipole mode at $4.140 \mathrm{GHz}$, and for no obvious reason we find a very strong signal around $4.210 \mathrm{GHz}$. All signals are seen to vary linearly with the beam position, i.e. they are 
indeed dipole modes. We performed bench-measurements on a standard ten foot structure to investigate the modespectrum. Fig. 3 shows VSWR measurements of the structure from both ports, demonstrating that roughly $\mathbf{5 0}$ modes (out of 86) can be accessed from the input coupler. Here the lowest mode was observed at $4.143 \mathrm{GHz}$ and no unusual structure was found around $4.210 \mathrm{GHz}$.

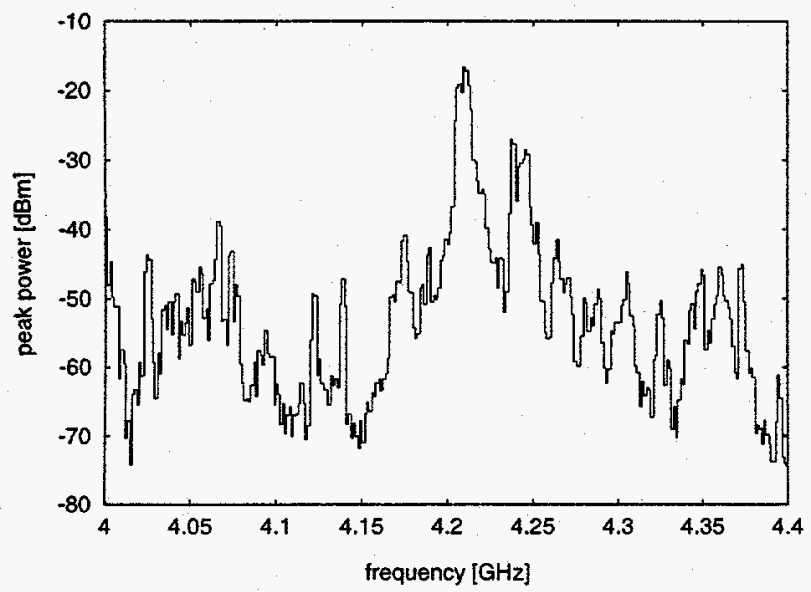

Figure 2: Typical beam excited dipole mode spectrum measured at a single structure (20-3c).

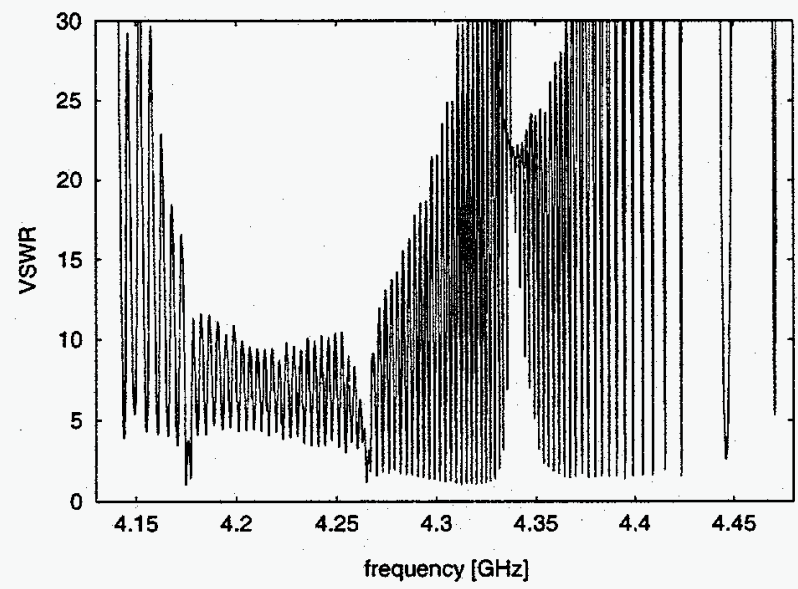

Figure 3: Standing wave ratio measurement on a ten foot structure. Left side from input end, right side output end.

\section{BEAM STEERING EXPERIMENTS}

In the simplest case we used gated ADC's to digitize the crystal detector video signals. The beam was steered in both planes to minimize the signals and to find the structure electrical center where minimal wakefield deflection should occur. This scheme was tried in sector 29 of the SLC linac were all klystron stations have been equipped with bandpass filters and crystal detectors. In this sector each klystron feeds four structures. In Fig. 4 we show the vertical beam positions at which the crystal detector signal was minimal, measured with BPM's. The data points exhibit a mostly positive offset of the order of $1 \mathrm{~mm}$ which is far outside the alignment tolerance of structures and BPM's. One possible explanation is that the signals are biased by $\mathrm{x}$ polarized fields, although this would unlikely shift all measurements to one side.

More information on beam-to-structure alignment can be extracted by a spectral analysis of the dipole mode signals. The beam is swept in steps transversely across the structure and a complete spectrum is recorded at each step. The resulting two-dimensional power spectrum as a function of frequency and position is then fit slicewise to a parabolic parametrization with respect to the position. As one of the fitparameters we obtain the minimum power position of the beam as a function of frequency. Because of the detuning of the structure the frequency of the signal provides a measure of the longitudinal position in the structure were it originated. The result of such a measurement is shown in Fig. 5. We find a smooth variation of $x_{\min }$ as a function of frequency. This method has been applied later successfully to perform a beam-based straightness measurement of an Xband structure [4].

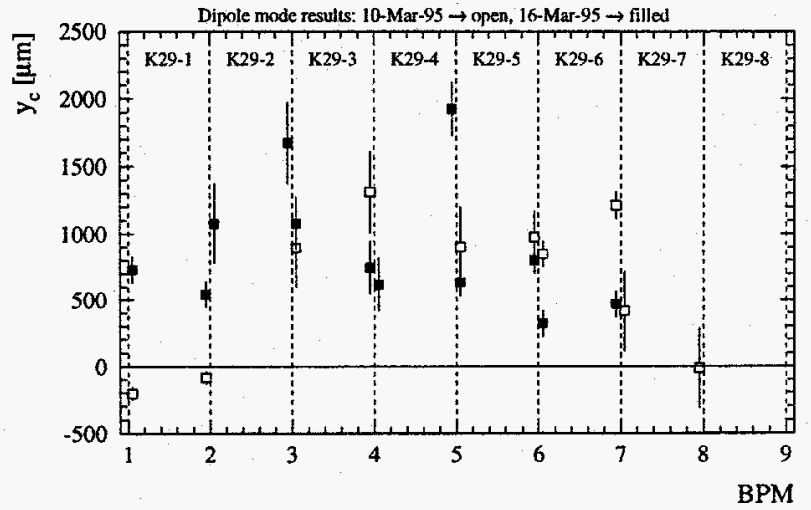

Figure 4: Measured vertical beam positions at 8 stations in sector 29 for minimum excitation of dipole modes in the corresponding structures.

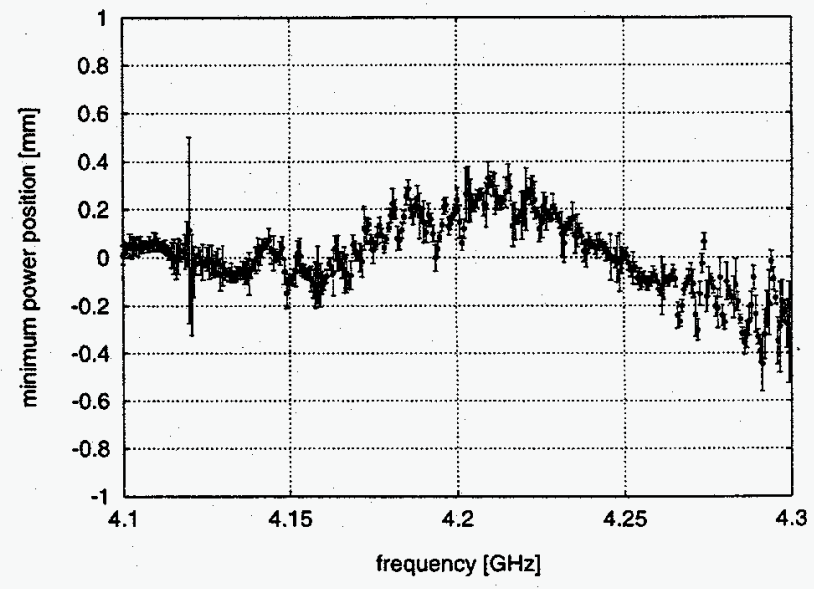

Figure 5: Minumum power positions in the horizontal plane as a function of frequency, obtained from a parallel beam scan at station $2-2$.

Finally, the most precise way to measure the beam position locally in a structure is to detect both amplitude and 
phase with respect to beam arrival time of the dipole mode signal at a certain frequency. This was achieved in our setup by using the spectrum analyzer as a receiver at a fixed frequency and analyzing the first stage IF signal $\left(f_{\mathrm{IF}}=\right.$ $310 \mathrm{MHz}$ ). For the phase measurement a broad band BPM signal was filtered around $4 \mathrm{GHz}$ and mixed with the spectrum analyzer LO signal as well. The resulting two IF signals were then digitized in a fast scope and their amplitudes and relative phases determined by fitting sine functions to them.

The measured signal can be interpreted as a superposition of a component that scales linearly with the beam position and another component at some arbitrary phase that stays constant. The position independent component originates from coupling to fields polarized in the other plane, or from another structure that is connected to the same waveguide network.

$$
\begin{aligned}
U(x, t)= & A\left(x-x_{0}\right) \cos (\omega t)+B \cos (\omega t-\Delta \phi) \\
= & \hat{U}(x) \sin (\omega t+\phi(x)) \\
\hat{U}(x)= & \left(A^{2}\left(x-x_{0}\right)^{2}+\right. \\
& \left.\quad+2 A B\left(x-x_{0}\right) \cos (\Delta \phi)+B^{2}\right)^{1 / 2} \\
\phi(x)= & \arctan \left(\frac{A\left(x-x_{0}\right)}{B \sin (\Delta \phi)}+\cot (\Delta \phi)\right)
\end{aligned}
$$

When the beam is moved transversely across the structure the amplitude $\hat{U}(x)$ reaches some minimum value, determined by the size of the out-of-phase component and grows again afterwards. In the same course the phase of the signal $\phi(x)$ shifts by $180^{\circ}$. A linearized beam position measurement can thus be obtained from the measured phase and amplitude by computing

$$
\hat{U}(x) \sin (\phi(x))=A\left(x-x_{0}\right)+B \cos (\Delta \phi)
$$

Note that the position independent signal component shifts the minimum power position by $-B \cos (\Delta \phi) / A$.

The linearized result of such a beam scan in the horizontal plane is shown in Fig. 6. This measurement has been performed at station 2-2 that feeds two structures. An orbit fit from fifteen BPM's has been used to determine the real beam position at the location of the two structures. From a correlation analysis of the linearized dipole mode signal with the BPM data we obtain a resolution of $12 \mu \mathrm{m}$ for our structure BPM.

\section{SUMMARY}

In summary we were able to detect beam induced microwave signals in the lowest dipole mode band in the SLC S-Band structures. Beam-to-structure alignment information was extracted in several ways. Using amplitude and phase detection a position measurement resolution of

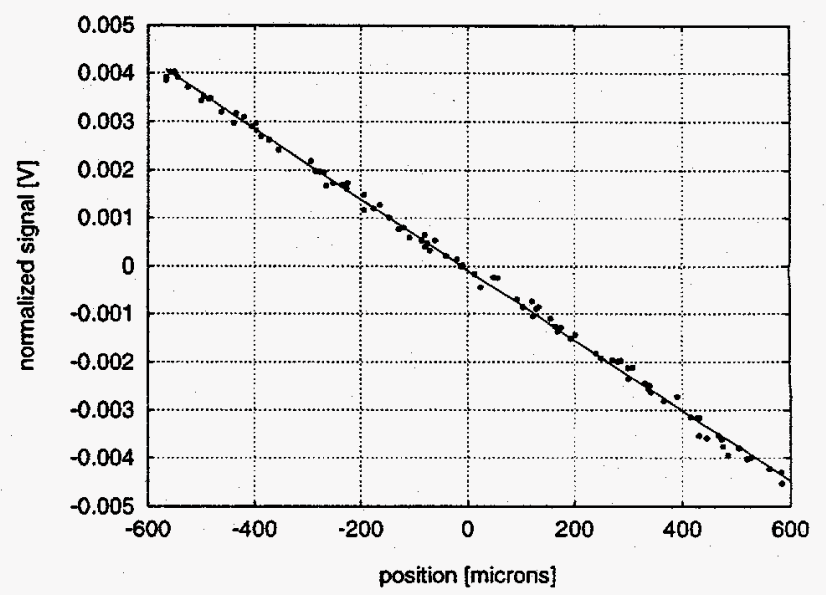

Figure 6: Linearized and signed amplitude of the dipole mode signal at station $2-2$, frequency $4.139 \mathrm{GHz}$, as a function of the horizontal beam position determined with BPM's. The solid line is a linear fit.

$12 \mu \mathrm{m}$ was demonstrated. However, there is a much larger uncertainty in the determination of the absolute center position in the structure. This uncertainty is believed to be caused by mainly two reasons:

1. The SLC structures have no dedicated HOM couplers that would allow one to separate horizontally and vertically polarized fields. Using the standard input coupler we measure a mixture of both signals which couples the response to offsets in both planes. Furthermore the input and output couplers introduce intrinsic asymmetries in the horizontal plane of the structure that might shift the electrical center for certain modes.

2. The detected dipole mode signals are a superposition from several structures which could introduce interference effects.

Despite the described problems, dipole mode detection can be useful if the signal is carefully minimized in multidimensional beam position/angle scans in both planes. The signal amplitude at the minimum of a one-dimensional scan can be used to assess the quality of the beam-to-structure alignment. As a first step the goal in the SLC is to identify badly misaligned structures which can be accomplished with the described methods. This work was supported by the Department of Energy, contract DE-AC03-76SF00515.

\section{REFERENCES}

[1] R.B. Neal et al., The Stanford Two-Mile Accelerator, W.A.Benjamin Inc. (1968)

[2] A.M. Vetter, J.L. Adamski, W.J. Gallagher, IEEE Trans. Nucl. Sci. NS-32, 2329 (1983)

[3] C. Peschkeet al, in Proceedings 5th European Particle Accelerator Conference, Sitges, Spain (1996)

[4] M. Seidel, these proceedings 